\title{
QUANTUM CURVES FOR SIMPLE HURWITZ NUMBERS OF AN ARBITRARY BASE CURVE
}

\author{
XIAOJUN LIU, MOTOHICO MULASE, AND ADAM SORKIN
}

\begin{abstract}
The generating functions of simple Hurwitz numbers of the projective line are known to satisfy many properties. They include a heat equation, the Eynard-Orantin topological recursion, an infinite-order differential equation called a quantum curve equation, and a Schrödinger like partial differential equation. In this paper we generalize these properties to simple Hurwitz numbers with an arbitrary base curve.
\end{abstract}

\section{Contents}

1. Introduction and the main results

2. A cut-and-join equation for simple Hurwitz numbers

3. The discrete Laplace transform

4. A Schrödinger equation

5. The heat equation and its consequences 10

6. The quantum curve $\quad 12$

7. Semi-classical limit 13

References

\section{INTRODUCTION AND THE MAIN RESULTS}

The purpose of this paper is to determine functional properties of various generating functions of simple Hurwitz numbers with an arbitrary fixed base curve $B$. We derive a partial differential equation for the Laplace transform of these Hurwitz numbers. The equation is completely analogous to the result of [28] for the usual simple Hurwitz numbers based on $\mathbb{P}^{1}$. We also obtain an infinite-order differential equation, or a quantum curve, for the case of an arbitrary base curve, that is parallel to the various Hurwitz problems studied in $\left[3,25,26\right.$, with the base curve $\mathbb{P}^{1}$ and its twisted version $\mathbb{P}^{1}[a]$.

Our main motivation is to examine whether the topological recursion of Eynard-Orantin [14, 15] and the existence of a quantum curve of [1, 7, 8, 9, 19] hold for the enumeration problem of simple Hurwitz numbers over an arbitrary curve $B$. Consider a holomorphic map $f: C \longrightarrow B$ of a non-singular algebraic curve $C$ onto a fixed base curve $B$. We choose a general point $0 \in B$ and fix it once for all. The quantity we are interested in this paper is the base $B$ Hurwitz number $H_{g, n}^{B}\left(\mu_{1}, \ldots, \mu_{n}\right)$, which counts the automorphism weighted number of the topological types of holomorphic maps $f$ of a genus $g$ domain curve with $n$ labeled preimages of $0 \in B$ of multiplicity $\left(\mu_{1}, \ldots, \mu_{n}\right) \in \mathbb{Z}_{+}^{n}$, such that $f$ is simply ramified

2000 Mathematics Subject Classification. Primary: 14H15, 14N35, 05C30, 11P21; Secondary: 81T30. 
other than these $n$ points. Define its discrete Laplace transform

$$
\begin{aligned}
F_{g, n}^{B}\left(x_{1}, \ldots, x_{n}\right) & =\sum_{\vec{\mu} \in \mathbb{Z}_{+}^{n}} H_{g, n}^{B}\left(\mu_{1}, \ldots, \mu_{n}\right) \prod_{i=1}^{n} e^{-w_{i} \mu_{i}} \\
& =\sum_{\vec{\mu} \in \mathbb{Z}_{+}^{n}} H_{g, n}^{B}\left(\mu_{1}, \ldots, \mu_{n}\right) \prod_{i=1}^{n} x_{i}^{\mu_{i}}, \quad x_{i}=e^{-w_{i}},
\end{aligned}
$$

which we call the free energy of type $(g, n)$. Since $\mathbb{P}^{1}$ does not cover the base curve $B$ of genus $g(B)>0$,

$$
F_{0, n}^{B}\left(\mu_{1}, \ldots, \mu_{n}\right)=0
$$

for any $n \geq 1$ and any value of $\left(\mu_{1}, \ldots, \mu_{n}\right) \in \mathbb{Z}_{+}^{n}$ in this case. Our main result is the following.

Theorem 1.1. For $2 g-2+n>0$, the free energies $F_{g, n}^{B}\left(x_{1}, \ldots, x_{n}\right)$ satisfy the following partial differential equation:

$$
\begin{aligned}
(2 g-2+n- & \left.(1-\chi(B)) \sum_{i=1}^{n} x_{i} \frac{\partial}{\partial x_{i}}\right) F_{g, n}^{B}\left(x_{[n]}\right) \\
= & \frac{1}{2} \sum_{i \neq j} \frac{x_{i} x_{j}}{x_{i}-x_{j}}\left(\frac{\partial}{\partial x_{i}} F_{g, n-1}^{B}\left(x_{[\hat{j}]}\right)-\frac{\partial}{\partial x_{j}} F_{g, n-1}^{B}\left(x_{[\hat{i}]}\right)\right) \\
& +\left.\frac{1}{2} \sum_{i=1}^{n} u_{1} \frac{\partial}{\partial u_{1}} u_{2} \frac{\partial}{\partial u_{2}}\right|_{u_{1}=u_{2}=x_{i}} \\
& {\left[F_{g-1, n+1}^{B}\left(u_{1}, u_{2}, x_{[\hat{i}]}\right)+\sum_{\substack{g_{1}+g_{2}=g \\
I \sqcup J=[\hat{i}]}} F_{g_{1},|I|+1}^{B}\left(u_{1}, x_{I}\right) F_{g_{2},|J|+1}^{B}\left(u_{2}, x_{J}\right)\right] . }
\end{aligned}
$$

Here $[n]=\{1, \ldots, n\}$ is an index set, $[\hat{i}]=[n] \backslash\{i\}$, and for any subset $I \subset[n]$, we denote $x_{I}=\left(x_{i}\right)_{i \in I}$. We denote by $\chi(B)=2-2 g(B)$ the Euler characteristic of the base curve $B$. Note that the complexity $2 g-2+n$ is reduced by 1 on the right-hand side of the recursion when $g(B) \geq 1$, similar to the Eynard-Orantin integral recursion of [14, 15].

Let us define the partition function of the base B Hurwitz numbers, as a holomorphic function in $x \in \mathbb{C}$ and $\hbar$ with $\operatorname{Re}(\hbar)<0$, by

$$
Z^{B}(x, \hbar)=\exp \left(\sum_{g=1}^{\infty} \sum_{n=1}^{\infty} \frac{1}{n !} \hbar^{2 g-2+n} F_{g, n}^{B}(x, x, \ldots, x)\right) .
$$

Then it satisfies a quantum curve like infinite-order differential equation

$$
\hbar x \frac{d}{d x}\left[1-\hbar^{1-\chi(B)} x e^{\hbar x \frac{d}{d x}}\left(\frac{d}{d x} x\right)^{1-\chi(B)}\right] Z^{B}(x, \hbar)=0 .
$$

If we introduce

$$
y=\hbar x \frac{d}{d x}
$$


and regard it as a commuting variable, then the total symbol of the above operator produces a Lambert curve

$$
x=y^{\chi(B)-1} e^{-y} .
$$

The partition function also satisfies a Schrödinger like partial differential equation

$$
\left[\frac{\partial}{\partial \hbar}-\frac{1}{2}\left(x \frac{\partial}{\partial x}\right)^{2}+\left(\frac{1}{2}-\frac{1-\chi(B)}{\hbar}\right) x \frac{\partial}{\partial x}\right] Z^{B}(x, \hbar)=0 .
$$

If we denote the above operators by $P$ and $Q$, respectively, then they satisfy a commutation relation:

$$
[P, Q]=-\frac{1}{\hbar} P \text {. }
$$

This shows that the system of equations (1.4) and (1.6) are compatible. Moreover, the partition function has the following simple expression

$$
Z^{B}(x, \hbar)=\sum_{m=0}^{\infty}(m !)^{1-\chi(B)} e^{\frac{1}{2} m(m-1) \hbar}\left(\hbar^{1-\chi(B)} x\right)^{m} .
$$

Although the parameter $\hbar$ is a formal deformation parameter, if we write $\hbar=2 \pi i \tau$, then

$$
Z^{B}(x, 2 \pi i \tau)=\sum_{m=0}^{\infty}(m !)^{1-\chi(B)} e^{\pi i m(m-1) \tau}\left((2 \pi i \tau)^{1-\chi(B)} x\right)^{m}
$$

is an entire function in $x$ for $\operatorname{Im}(\tau)>0$.

Remark 1.2. We note that the exact same formulas (1.2), (1.5), (1.6), and (1.8) hold for the case of an orbifold base $B=\mathbb{P}^{1}[a], a>0$, if we evaluate $\chi(B)=1+\frac{1}{a}$, interpret the sum in (1.8) as running over non-negative multiples $a m$ of $a$, and replace $(a m)$ ! by $(a m) ! \longmapsto\left(m ! a^{m}\right)^{a}$. Since the orbifold case requires a different set of preparations (see $[3,10,25]$ ), we will report the generalization to the case of higher-genus twisted curve as a base elsewhere.

When the base curve $B$ is an elliptic curve $E$, a special case of simple Hurwitz numbers exhibits a quasi-modular property. Dijkgraaf [6] considered a generating function

$$
F_{g}(q)=\sum_{n=1}^{\infty} \frac{1}{n !} H_{g, n}^{E}(1,1, \ldots, 1) q^{n}
$$

for $g>1$, and

$$
F_{1}(q)=-\frac{1}{24} \log q+\sum_{n=1}^{\infty} \frac{1}{n !} H_{1, n}^{E}(1,1, \ldots, 1) q^{n} .
$$

The significant fact here is that $F_{g}(q)$ for $g>1$ is a quasi-modular form [6, 21]. The relation between the free energies of type $(g, n)$ for all $n$ and $F_{g}(q)$ is given by

$$
F_{g}(q)=\lim _{\lambda \rightarrow 0} \sum_{n=1}^{\infty} \frac{1}{n !} \frac{1}{\lambda^{n}} F_{g, n}^{E}(\lambda q, \lambda q, \ldots, \lambda q) .
$$

To motivate the present work, let us recall the corresponding counting problem of simple Hurwitz number $H_{g, n}^{\mathbb{P}^{1}}\left(\mu_{1}, \ldots, \mu_{n}\right)$ for a pointed projective line $\left(\mathbb{P}^{1}, \infty\right)$, which has a long history since Hurwitz [20]. Its modern interest is due, among other things, to its rich functional properties [16, 17, 22, 32, 33], its relation with linear Hodge integrals on $\overline{\mathcal{M}}_{g, n}$ 
[12, 18, 30, and the Bouchard-Mariño conjecture [5] and its solutions [2, 13, 28]. The theorem established in [13, 28] shows that the discrete Laplace transform

$$
F_{g, n}^{\mathbb{P}^{1}}\left(x_{1}, \ldots, x_{n}\right)=\sum_{\vec{\mu} \in \mathbb{Z}_{+}^{n}} H_{g, n}^{\mathbb{P}^{1}}\left(\mu_{1}, \ldots, \mu_{n}\right) \prod_{i=1}^{n} x_{i}^{\mu_{i}}
$$

satisfies an integral recursion formula that was originally proposed by Eynard and Orantin [14, as conjectured in [5]. The input curve for the recursion, the spectral curve, is shown to be the original Lambert curve

$$
x=y e^{-y} .
$$

Then in [26, 34] it is discovered that there is a quantum curve of [1, 7, 8, 9, 19], which is a generator of the holonomic system that characterizes the partition function of the simple Hurwitz numbers of $\mathbb{P}^{1}$. The partition function for $B=\mathbb{P}^{1}$ is

$$
Z^{\mathbb{P}^{1}}(x, \hbar)=\exp \left(\sum_{g=0}^{\infty} \sum_{n=1}^{\infty} \frac{1}{n !} \hbar^{2 g-2+n} F_{g, n}^{\mathbb{P}^{1}}(x, x, \ldots, x)\right) .
$$

It is established in [26] that the $\mathbb{P}^{1}$ partition function has an expression

$$
Z^{\mathbb{P}^{1}}(x, \hbar)=\sum_{m=0}^{\infty} \frac{1}{m !} e^{\frac{1}{2} m(m-1) \hbar}\left(\frac{x}{\hbar}\right)^{m},
$$

and that it satisfies two equations:

$$
\begin{aligned}
& {\left[\hbar x \frac{\partial}{\partial x}-x e^{\hbar x \frac{\partial}{\partial x}}\right] Z^{\mathbb{P}^{1}}(x, \hbar)=0} \\
& {\left[\frac{\partial}{\partial \hbar}-\frac{1}{2}\left(x \frac{\partial}{\partial x}\right)^{2}+\left(\frac{1}{2}+\frac{1}{\hbar}\right) x \frac{\partial}{\partial x}\right] Z^{\mathbb{P}^{1}}(x, \hbar)=0 .}
\end{aligned}
$$

If we denote the above operators as $P_{1}$ and $Q_{1}$, respectively, then they satisfy a commutation relation

$$
\left[P_{1}, Q_{1}\right]=-\frac{1}{\hbar} P_{1}
$$

The semi-classical limit of each of the equations (1.16) and (1.17) recovers the Lambert curve (1.14), as shown in [26]. Then from this curve as the spectral curve, and using the functions $x$ and $y$ on it, the Eynard-Orantin integral recursion [14] determines the differential forms

$$
d_{1} d_{2} \cdots d_{n} F_{g, n}^{\mathbb{P}^{1}}
$$

defined on $\Sigma^{n}$ for all $(g, n)$, where $\Sigma$ is the Lambert curve given by (1.14). It is a simple consequence of the results in [13, 26, 28] that the differential form (1.19) uniquely recovers the primitive $F_{g, n}^{\mathbb{P}^{1}}$ as a function in $\left(y_{1}, \ldots, y_{n}\right) \in \Sigma^{n}$. From this point of view, the generating function $F_{g, n}^{\mathbb{P}^{1}}\left(x\left(y_{1}\right), \ldots, x\left(y_{n}\right)\right)$ is completely determined by the equation (1.16) or (1.17), where $x$ as a function in $y$ is also given by (1.14).

Our motivating question is, do the similar properties hold when we consider the simple Hurwitz numbers with an arbitrary curve as the base? Since the genus 0 base $B$ Hurwitz numbers $H_{0, n}^{E}\left(\mu_{1}, \ldots, \mu_{n}\right)$ do not exist for a base curve $B$ with $g(B)>0$, the philosophy of [1] does not produce any spectral curve of the Eynard-Orantin integral recursion for this counting problem. Yet we have a topological recursion (1.2) in the form of a partial differential equation. We note the straightforward generalizations (1.4), (1.6), (1.8), and 
(1.5), that reduce to the $\mathbb{P}^{1}$ case (1.16), (1.17), (1.15), and (1.14), respectively, when $\chi(B)=$ 2 .

The paper is organize as follows. In Section 2, we derive the cut-and-join equation of simple Hurwitz numbers with an arbitrary base curve. Then in Section 3, we consider the Laplace transform of the cut-and-join equation, which is exactly (1.2). Using this result we derive the Schrödinger equation (1.6) in Section 4. Section 5 is devoted to explaining the heat equation expression of the cut-and-join equation [16, 22, 33] and deriving its consequences. In Section [6 we first prove the expansion (1.8), derive the quantum curve (1.4), and then establish the commutator relation (1.7). In the final section we deduce the Lambert curve (1.5) as the semi-classical limit.

\section{A CUT-And-Join EQUATion For Simple HuRWitz NUmbers}

The enumeration problem we consider in this paper is the number of topological types of holomorphic maps $f: C \longrightarrow B$ from a nonsingular curve $C$ of genus $g$ to a fixed base curve $B$, with an arbitrary ramification over one point on $B$ and simple ramification at all other critical points. Let us denote by $0 \in B$ a general point arbitrarily chosen and fixed. Such a homomorphic map $f$ is referred to as a simple Hurwitz cover of $B$. We label each inverse image of $0 \in B$ via $f$, and denote by $\left(\mu_{1}, \ldots, \mu_{n}\right) \in \mathbb{Z}_{+}^{n}$ the degrees of $f$ at each inverse image of 0 . The base $B$ Hurwitz number $H_{g, n}^{B}\left(\mu_{1}, \ldots \mu_{n}\right)$ counts the automorphism weighted number of the topological types of simple Hurwitz covers.

The degree of the map $f$ is given by

$$
d=|\mu|=\sum_{i=1}^{n} \mu_{i} .
$$

Here use the notation $|\mu|$, borrowing from the convention in the theory symmetric functions. The Riemann-Hurwitz formula tells us that there are

$$
r=r(g, \mu)=2 g-2+n-d(2 g(B)-1)
$$

simple ramification points. Instead of counting the topological types of $f$, we can fix $r$ simple branch points on $B$ in general position other than $0 \in B$ and count the automorphism weighted holomorphic maps $f$. We note that (2.1) and (2.2) imply a condition for the degree and the genus for a base curve with $g(B) \geq 1$ :

$$
\begin{aligned}
& n(2 g(B)-1) \leq d(2 g(B)-1) \leq 2 g-2+n, \\
& n(g(B)-1) \leq g-1 .
\end{aligned}
$$

In particular, $H_{g, n}(\vec{\mu})=0$ for any $n \geq 1$ if $g<g(B)$. This poses a sharp contrast to the Gromov-Witten theory of curves [31].

The map $f: C \longrightarrow B$ of degree $d$ is determined by a monodromy representation

$$
\rho \in \operatorname{Hom}\left(\pi_{i}(B \backslash\{0,1, \ldots, r,\}), S_{d}\right)
$$

of the fundamental group of an $(r+1)$-punctured curve into the symmetric group of $d$ letters. Here $\{1, \ldots, r\} \subset B$ is the label of $r$ simple branched points on $B$ chosen in general position. Let $\gamma_{j}, j=0, \ldots, r$, be a simple loop around each point $j \in B$. Then a simple Hurwitz cover is constructed by assigning each $\gamma_{j}$ for $j \geq 1$ to a transposition and $\gamma_{0}$ to a product of $n$ disjoint cycles of length $\mu_{1}, \cdots \mu_{n}$, subject to the commutator relation

$$
\rho\left(\gamma_{1}\right) \cdots \rho\left(\gamma_{r}\right) \rho\left(\gamma_{0}\right)=\left[\alpha_{1}, \beta_{1}\right] \cdots\left[\alpha_{g(B)}, \beta_{g(B)}\right]
$$

where $\alpha_{i}, \beta_{i} \in S_{d}$ are some elements in the permutation group. 
The cut-and-join equation [16, 17, 20, 32] is the result of an analysis of what happens when we multiply a transposition $\rho\left(\gamma_{r}\right)$ to a product of disjoint cycles $\rho\left(\gamma_{0}\right)$. Since the fundamental group of the base curve does not make any effect on this multiplication, the exact same formula for $H_{g, n}^{B}(\vec{\mu})$ holds, as for the simple Hurwitz numbers for $\mathbb{P}^{1}$. The only difference is the number $r$ of simple ramification points.

Proposition 2.1 (The cut-and-join equation). For all $g \geq 0, n \geq 1$, and $\left(\mu_{1}, \ldots, \mu_{n}\right) \in \mathbb{Z}_{+}^{n}$ subject to

$$
2 g-2+n-(2 g(B)-1) \sum_{i=1}^{n} \mu_{i}>0,
$$

the simple Hurwitz numbers $H_{g, n}^{B}\left(\mu_{1}, \ldots, \mu_{n}\right)$ of degree $d$ satisfy the following equation:

$$
\begin{gathered}
(2 g-2+n-(2 g(B)-1) d) H_{g, n}^{B}\left(\mu_{[n]}\right)=\frac{1}{2} \sum_{i \neq j}\left(\mu_{i}+\mu_{j}\right) H_{g, n-1}^{B}\left(\mu_{i}+\mu_{j}, \mu_{[\hat{i}, \hat{j}]}\right) \\
+\frac{1}{2} \sum_{i=1}^{n} \sum_{\alpha+\beta=\mu_{i}} \alpha \beta\left(H_{g-1, n+1}^{B}\left(\alpha, \beta, \mu_{[\hat{i}]}\right)+\sum_{\substack{g_{1}+g_{2}=g \\
I \sqcup J=[\hat{i}]}} H_{g_{1},|I|+1}^{B}\left(\alpha, \mu_{I}\right) H_{g_{2},|J|+1}^{B}\left(\beta, \mu_{J}\right)\right) .
\end{gathered}
$$

Here and throughout the paper we use the following notational convention. $[n]=\{1, \ldots, n\}$ is an index set, and $[\hat{i}]=[n] \backslash\{i\}$, etc. For a subset $I \subset[n], \mu_{I}=\left(\mu_{i}\right)_{i \in I}$.

Remark 2.2. Unlike the case of $\mathbb{P}^{1}$, the cut-and-join equation does not determine all values of simple Hurwitz numbers. When the degree $d$ takes its maximum value $\frac{2 g-2+n}{2 g(B)-1}$, the equation gives a trivial equality $0=0$.

The genus 1 simple Hurwitz numbers based on an elliptic curve $B=E$ are easy to calculate. From (2.3) we have $n=d$ and $r=0$ when $g=g(B)=1$. Therefore, the covering is unramified. If we allow disconnected domain, then the total number is equal to the number of partitions

$$
p(d)=\left|\operatorname{Hom}\left(\pi_{1}(E), S_{d}\right) / / S_{d}\right|
$$

of degree $d$ (see for example, [6]). Let

$$
\phi(q)=\prod_{m=1}^{\infty}\left(1-q^{m}\right)
$$

be the Euler function. Then we have

$$
\sum_{n=1}^{\infty} \frac{1}{n !} H_{1, n}^{E}(1, \ldots, 1) q^{n}=-\log \phi(q)=\sum_{n=1}^{\infty}\left(\sum_{m \mid n} \frac{1}{m}\right) q^{n},
$$

hence

$$
H_{1, n}^{E}(1, \ldots, 1)=n ! \sum_{m \mid n} \frac{1}{m}=(n-1) ! \sigma(n),
$$

where $\sigma$ is the sum of divisors function. This is an integer sequence, and its first ten terms are $1,3,8,42,144,1440,5760,75600,524160,6531840$. This sequence has many interesting properties (see OEIS, A038048).

More generally, let us consider the case when the equality holds in (2.4). We need this analysis in Section 4. Because of (2.3),$g-1=n(g(B)-1)$ implies $d=n$ and $r=0$, hence 
the covering $f: C \longrightarrow B$ that is counted is totally unramified. Here again the number of disconnected unramified coverings is given by the classical dimension formula (see for example [27] for elementary derivations):

$$
\left|\operatorname{Hom}\left(\pi_{1}(B), S_{d}\right) / / S_{d}\right|=\sum_{\lambda \vdash d}\left(\frac{\operatorname{dim} \lambda}{d !}\right)^{\chi(B)} .
$$

Here $\lambda \vdash d$ parametrizes irreducible representations of $S_{d}$, and $\operatorname{dim} \lambda$ is its dimension.

\section{THE DISCRETE LAPLACE TRANSFORM}

The discrete Laplace transform $F_{g, n}^{B}\left(x_{1}, \ldots, x_{n}\right)$ of (1.1) is a polynomial of degree $\frac{2 g-2+n}{2 g(B)-1}$ with the lowest degree term $H_{g, n}^{B}(1,1, \ldots, 1) x_{1} \cdots x_{n}$. The following proposition is an analogue of the case of simple Hurwitz numbers of $\mathbb{P}^{1}$, and the proof is exactly the same as that of [3, Lemma 4.1].

Proposition 3.1. The discrete Laplace transform of the cut-and-join equation (2.6) is precisely the partial differential equation (1.2).

Reflecting Remark 2.2, the differential equation (1.2) alone does not determine free energies $F_{g, n}^{B}\left(x_{1}, \ldots, x_{n}\right)$. This is because every homogeneous function of degree $\frac{2 g-2+n}{2 g(B)-1}$ is in the kernel of the Euler differential operator

$$
2 g-2+n-(2 g(B)-1) \sum_{i=1}^{n} x_{i} \frac{\partial}{\partial x_{i}}
$$

on the left-hand side of (1.2). Thus the highest degree terms of free energies are not determined by this differential equation. We will determine the homogeneous highest degree terms of the free energies in a different method in Section 5 .

The genus 1 elliptic Hurwitz numbers (2.7) yields

$$
F_{1, n}^{E}\left(x_{1}, \ldots, x_{n}\right)=\left(n ! \sum_{m \mid n} \frac{1}{m}\right) x_{1} \cdots x_{n} .
$$

\section{A SchröDinger EQUATION}

In this section we prove (1.6), provided that $g(B)>0$. We remark here that for $g(B)=0$, the proof is rather different [26], yet the same formula holds.

Theorem 4.1. The partition function (1.3) satisfies the Schrödinger-type equation (1.6):

$$
\left[\frac{\partial}{\partial \hbar}-\frac{1}{2}\left(x \frac{\partial}{\partial x}\right)^{2}+\left(\frac{1}{2}-\frac{2 g(B)-1}{\hbar}\right) x \frac{\partial}{\partial x}\right] Z^{B}(x, \hbar)=0 .
$$


Proof. We use the same method of the proof of [26, Theorem 5.1, Theorem 5.3, Appendix A]. When (2.3) holds, the diagonal evaluation of (1.2) yields

$$
\begin{aligned}
& \left(2 g-2+n-(2 g(B)-1) x \frac{d}{d x}\right) F_{g, n}^{B}(x, \ldots, x) \\
& =\left.\frac{n(n-1)}{2} x^{2} \frac{\partial^{2}}{\partial u^{2}}\right|_{u=x} F_{g, n-1}^{B}(u, x, \ldots, x) \\
& +\left.\frac{n}{2} x^{2} \frac{\partial^{2}}{\partial u_{1} \partial u_{2}}\right|_{u_{1}=u_{2}=x} F_{g-1, n+1}^{B}\left(u_{1}, u_{2}, x, \ldots, x\right) \\
& +\frac{n !}{2} \sum_{\substack{g_{1}+g_{2}=g \\
n_{1}+n_{2}=n-1}} \frac{x^{2}}{\left(n_{1}+1\right) !\left(n_{2}+1\right) !} \frac{d}{d x} F_{g_{1}, n_{1}+1}^{B}(x, \ldots, x) \cdot \frac{d}{d x} F_{g_{2}, n_{2}+1}^{B}(x, \ldots, x) .
\end{aligned}
$$

For $m \geq 2 g(B)$, let us define

$$
S_{m}(x):=\sum_{2 g-2+n=m-1} \frac{1}{n !} F_{g, n}^{B}(x, \ldots, x) .
$$

Note that $S_{m}$ contains a contribution from domain curves with $g-1=n(g(B)-1)$, or equivalently, $d=n$ and $r=0$, for which the cut-and-join equation is not valid. Therefore, when we deduce an equation for $S_{m}$ 's, we need to remove these boundary terms from the equation. Now from (4.1) we obtain

$$
\begin{aligned}
& \left(m-(2 g(B)-1) x \frac{d}{d x}\right) S_{m+1}(x) \\
& -\frac{1}{2} x^{2} \frac{d^{2}}{d x^{2}} S_{m}(x)-\frac{1}{2} \sum_{m_{1}+m_{2}=m+1} x \frac{d}{d x} S_{m_{1}}(x) \cdot x \frac{d}{d x} S_{m_{2}}(x) \\
& =\sum_{\substack{2 g-2+n=m \\
g-1=n(g(B)-1)}} \frac{1}{n !}\left(m-(2 g(B)-1) x \frac{d}{d x}\right) F_{g, n}^{B}(x, \ldots, x) \\
& -\left.\frac{1}{2} \sum_{\substack{2 g-2+n=m-1 \\
g-1=(n+1)(g(B)-1)}} \frac{1}{(n-1) !} x^{2} \frac{\partial^{2}}{\partial u^{2}}\right|_{u=x} F_{g, n}^{B}(u, x, \ldots, x) \\
& -\left.\frac{1}{2} \sum_{\substack{2 g-2+n=m-1 \\
g=(n-1)(g(B)-1)}} \frac{1}{(n-2) !} x^{2} \frac{\partial^{2}}{\partial u_{1} \partial u_{2}}\right|_{u_{1}=u_{2}=x} F_{g, n}^{B}\left(u_{1}, u_{2}, x, \ldots, x\right) \\
& -\frac{1}{2} \sum_{\substack{2 g-2+n=m \\
g-1=n(g(B)-1)}} \sum_{\substack{g_{1}+g_{2}=g \\
n_{1}+n_{2}=n+1}} \frac{x^{2}}{n_{1} ! n_{2} !}\left(\frac{d}{d x} F_{g_{1}, n_{1}}^{B}(x, \ldots, x)\right)\left(\frac{d}{d x} F_{g_{2}, n_{2}}^{B}(x, \ldots, x)\right) .
\end{aligned}
$$

We note here that in the boundary contribution $g-1=n(g(B)-1)$, since $n=d$, the free energies are single monomials proportional to $x_{1} x_{2} \cdots x_{n}$. Let us look at the right-hand side of (4.2). The first line of the right-hand side is 0 because $F_{g, n}^{B}(x, \ldots, x)=H_{g, n}^{B}(1, \ldots, 1) x^{n}$, and $m=2 g-2+n=n(2 g(B)-1)$, since $r=0$. The second line is also 0 , because $F_{g, n}^{B}(u, x, \ldots, x)$ is linear in $u$.

The third summation on the right-hand side is empty, because we need $n(g(B)-1) \leq$ $g-1=(n-1)(g(B)-1)-1$, which does not happen. Similarly, the fourth line summation 
is also empty, because we are requiring $g=g_{1}+g_{2}, n+1=n_{1}+n_{2}$, and

$$
\begin{aligned}
n_{1}(g(B)-1) & \leq g_{1}-1 \\
n_{2}(g(B)-1) & \leq g_{2}-1 \\
n(g(B)-1) & =g-1 .
\end{aligned}
$$

We have thus obtained a recursion equation

$$
\begin{aligned}
\left(m-(2 g(B)-1) x \frac{d}{d x}\right) & S_{m+1}(x) \\
= & \frac{1}{2} x^{2} \frac{d^{2}}{d x^{2}} S_{m}(x)+\frac{1}{2} \sum_{m_{1}+m_{2}=m+1} x \frac{d}{d x} S_{m_{1}}(x) \cdot x \frac{d}{d x} S_{m_{2}}(x) .
\end{aligned}
$$

In terms of the generating function

$$
F(x, \hbar)=\sum_{m=1}^{\infty} \hbar^{m-1} S_{m}(x),
$$

(4.3) becomes

$$
\left(\frac{\partial}{\partial \hbar}-\frac{2 g(B)-1}{\hbar} x \frac{d}{d x}\right) F(x, \hbar)=\frac{1}{2} x^{2} \frac{d^{2}}{d x^{2}} F(x, \hbar)+\frac{1}{2}\left(x \frac{d}{d x} F(x, \hbar)\right)^{2} .
$$

Since $Z^{B}(x, \hbar)=\exp F(x, \hbar)$, (1.6) follows directly from (4.4). This completes the proof.

Using the Schrödinger equation (1.6), we can determine the form of the solution $Z^{B}(x, \hbar)$.

Lemma 4.2. The partition function has the following expansion:

$$
Z^{B}(x, \hbar)=\sum_{m=0}^{\infty} c_{m} e^{\frac{1}{2} m(m-1) \hbar}\left(\hbar^{1-\chi(B)} x\right)^{m},
$$

where $c_{m}$ is a constant.

Proof. The partition function $Z^{B}(x, \hbar)$ of (1.3) is a formal power series in $x$ and $\hbar$. Thus it has an expansion of the form

$$
Z^{B}(x, \hbar)=\sum_{m=0}^{\infty} c_{m} f_{m}(\hbar) x^{m}
$$

with $f_{m}(\hbar) \in \mathbb{C}[[\hbar]]$. Then the Schrödinger equation (1.6) yields an ordinary differential equation

$$
f_{m}^{\prime}=\left(\frac{1}{2} m(m-1)+m \frac{1-\chi(B)}{\hbar}\right) f_{m}
$$

whose solution is

$$
f_{m}(\hbar)=c e^{\frac{1}{2} m(m-1) \hbar} \hbar^{m(1-\chi(B))} .
$$

This completes the proof. 


\section{The heat EQUation AND its CONSEQUences}

As we have seen in Section 4, the Schrödinger equation (1.6) determines the solution $Z^{B}(x, \hbar)$ only up to the form (4.5) . To determine the coefficients, we need another technique.

In this section we use a heat equation technique of [22]. First we remark that the cutand-join equation gives rise to a heat equation for another generating function of base $B$ Hurwitz numbers. To determine a solution of a heat equation, we need to identify the initial value. We will show that the initial condition exactly corresponds to determining the highest degree terms of $F_{g, n}^{B}\left(x_{1}, \ldots, x_{n}\right)$. The exponential generating function of these highest degree terms can be determined by a character formula of [31. Thus we obtain the unique solution of the heat equation, which in turn gives all base $B$ Hurwitz numbers.

The generating function for base $B$ Hurwitz numbers we consider is

$$
\begin{aligned}
\mathbf{H}(t, \mathbf{p}) & =\sum_{g=0}^{\infty} \sum_{n=1}^{\infty} \mathbf{H}_{g, n}(t, \mathbf{p}), \\
\mathbf{H}_{g, n}(t, \mathbf{p}) & =\frac{1}{n !} \sum_{\vec{\mu} \in \mathbb{Z}_{+}^{n}} H_{g, n}^{B}(\vec{\mu}) \mathbf{p}_{\mu} t^{r(g, \mu)},
\end{aligned}
$$

where $r(g, \mu)=2 g-2+n-|\mu|(1-\chi(B))$ is the number of simple ramification points, and

$\mathbf{p}_{\mu}=p_{\mu_{1}} \cdots p_{\mu_{n}}$. The same argument of [16, 22, 23, 29, 33] shows that $e^{\mathbf{H}(t, \mathbf{p})}$ satisfies a heat equation, that is obtained from the cut-and-join equation. For a partition $\mu=\left(\mu_{1} \geq\right.$ $\left.\mu_{2} \geq \cdots\right)$ of a finite length $\ell(\mu)$, we define the shifted power-sum function by

$$
\mathbf{p}_{r}[\mu]:=\sum_{i=1}^{\infty}\left[\left(\mu_{i}-i+\frac{1}{2}\right)^{r}-\left(-i+\frac{1}{2}\right)^{r}\right] .
$$

This is a finite sum of $\ell(\mu)$ terms. Then we have [16, 33]

$$
\sum_{i, j \geq 1}\left((i+j) p_{i} p_{j} \frac{\partial}{\partial p_{i+j}}+i j p_{i+j} \frac{\partial^{2}}{\partial p_{i} \partial p_{j}}\right) s_{\mu}(\mathbf{p})=\mathbf{p}_{2}[\mu] \cdot s_{\mu}(\mathbf{p}),
$$

where $s_{\mu}(\mathbf{p})$ is the Schur function defined by

$$
s_{\mu}(\mathbf{p})=\sum_{|\lambda|=|\mu|} \frac{\chi_{\mu}(\lambda)}{z_{\lambda}} \mathbf{p}_{\lambda}, \quad z_{\mu}=\prod_{i=1}^{\ell(\mu)} m_{i} ! i^{m_{i}},
$$

$m_{i}=$ the number of parts in $\mu$ of length $i$, and $\chi_{\mu}(\lambda)$ is the value of the irreducible character of the representation $\mu$ of the symmetric group evaluated at the conjugacy class $\lambda$. Let us denote the cut-and-join operator by

$$
\triangle=\frac{1}{2} \sum_{i, j \geq 1}\left((i+j) p_{i} p_{j} \frac{\partial}{\partial p_{i+j}}+i j p_{i+j} \frac{\partial^{2}}{\partial p_{i} \partial p_{j}}\right) .
$$

Then Schur functions are eigenfunctions of the operator:

$$
\triangle s_{\mu}(\mathbf{p})=\frac{1}{2} \mathbf{p}_{2}[\mu] \cdot s_{\mu}(\mathbf{p}),
$$

and the cut-and-join equation of simple Hurwitz numbers (2.6) yields a heat equation

$$
\frac{\partial}{\partial t} e^{\mathbf{H}(t, \mathbf{p})}=\triangle e^{\mathbf{H}(t, \mathbf{p})} .
$$


We can solve a heat equation by the method of eigenfunction expansion. Thus we have an expression

$$
e^{\mathbf{H}(t, \mathbf{p})}=\sum_{\mu} a_{\mu} s_{\mu}(\mathbf{p}) e^{\frac{1}{2} \mathbf{p}_{2}[\mu] t}
$$

for a constant $a_{\mu}$ associated with every partition $\mu$. These constants are determined by the initial value $t=0$. From (5.2) we see that the initial value comes from the cases when $r(g, \mu)=0$.

First we note that (2.2) implies that simple Hurwitz numbers with $r=0$ correspond to the case with only 1 branched point on the base curve $B$. If we allow disconnected domain curves to cover $B$, then the number of coverings of degree $d$ with a prescribed ramification data given by a partition $\mu \vdash d$ over $0 \in B$ with no other ramification points is easy to calculate. Let us denote by $H_{d}^{B \bullet}(\mu)$ such a number, where we do not label the inverse images of $0 \in B$ this time. Then from [31, Eq.(0.10)] we obtain

$$
H_{d}^{B \bullet}(\mu)=\sum_{\lambda \vdash d}\left(\frac{\operatorname{dim} \lambda}{d !}\right)^{\chi(B)}\left|C_{\mu}\right| \frac{\chi_{\lambda}(\mu)}{\operatorname{dim} \lambda} .
$$

Here $C_{\mu}$ is the conjugacy class of the permutation group $S_{d}$ determined by the cycle type $\mu$, a partition $\lambda \vdash d$ is a label of an irreducible representation of $S_{d}$, and $\operatorname{dim} \lambda$ is its dimension. The cardinality of the conjugacy class is given by

$$
\left|C_{\mu}\right|=\frac{d !}{\prod_{i} m_{i} ! i^{m_{i}}}=\frac{d !}{z_{\mu}} .
$$

The generating function of these disconnected simple Hurwitz numbers can be calculated, appealing to (5.5) and (5.9), as follows:

$$
\begin{aligned}
\sum_{d=1}^{\infty} \sum_{\mu \vdash d} H_{d}^{B \bullet}(\mu) \mathbf{p}_{\mu} & =\sum_{d=1}^{\infty} \sum_{\mu \vdash d} \sum_{\lambda \vdash d}\left(\frac{\operatorname{dim} \lambda}{d !}\right)^{\chi(B)}\left|C_{\mu}\right| \frac{\chi_{\lambda}(\mu)}{\operatorname{dim} \lambda} \mathbf{p}_{\mu} \\
& =\sum_{d=1}^{\infty} \sum_{\lambda \vdash d} \frac{d !}{\operatorname{dim} \lambda}\left(\frac{\operatorname{dim} \lambda}{d !}\right)^{\chi(B)} \sum_{\mu \vdash d} \frac{\chi_{\lambda}(\mu)}{z_{\mu}} \mathbf{p}_{\mu} \\
& =\sum_{\lambda}\left(\frac{|\lambda| !}{\operatorname{dim} \lambda}\right)^{1-\chi(B)} s_{\lambda}(\mathbf{p}),
\end{aligned}
$$

where the last sum runs over all partitions $\lambda$.

Note that $e^{\mathbf{H}(t, \mathbf{p})}$ is the generating function of simple Hurwitz numbers allowing disconnected domain curves. Therefore, the initial value $e^{\mathbf{H}(0, \mathbf{p})}$ counts the disconnected base $B$ Hurwitz numbers with only one branched point at $0 \in B$. In other words, we have determined the initial condition by (5.10). The result is

$$
e^{\mathbf{H}(0, \mathbf{p})}=\sum_{\mu}\left(\frac{|\mu| !}{\operatorname{dim} \mu}\right)^{1-\chi(B)} s_{\mu}(\mathbf{p})=\sum_{\mu} a_{\mu} s_{\mu}(\mathbf{p}) .
$$

Since Schur functions are linear basis for symmetric functions, we establish the following.

Theorem 5.1. The exponential generating function of the base B simple Hurwitz numbers is given by

$$
e^{\mathbf{H}(t, \mathbf{p})}=\sum_{\mu}\left(\frac{|\mu| !}{\operatorname{dim} \mu}\right)^{1-\chi(B)} s_{\mu}(\mathbf{p}) e^{\frac{1}{2} \mathbf{p}_{2}[\mu] t} .
$$




\section{The QUANTUM CURVE}

As noted in [26], the diagonal specialization $F_{g, n}^{B}(x, x, \ldots, x)$ corresponds to substituting the power-sum symmetric function

$$
p_{j}=x_{1}^{j}+x_{2}^{j}+x_{3}^{j}+\cdots
$$

by its principal specialization $p_{j}=x^{j}$. More precisely, we have the following.

Lemma 6.1. Let us define the principal specialization of the power-sum symmetric functions by

$$
\begin{aligned}
p_{j}(s) & =\left(s^{1-\chi(B)} x\right)^{j} \\
\mathbf{p}_{\mu}(s) & =p_{\mu_{1}}(s) \cdots p_{\mu_{n}}(s)=\left(s^{1-\chi(B)} x\right)^{|\mu|}
\end{aligned}
$$

Then we have

$$
Z^{B}(x, \hbar)=e^{\mathbf{H}(\hbar, \mathbf{p}(\hbar))} .
$$

Proof. We have

$$
\begin{aligned}
\mathbf{H}(\hbar, \mathbf{p}(\hbar))=\sum_{g, n \geq 1} \sum_{\mu \in \mathbb{Z}_{+}^{n}} \frac{1}{n !} \hbar^{2 g-2+n-|\mu|(1-\chi(B))} H_{g, n}^{B}\left(\mu_{1}, \ldots, \mu_{n}\right) \mathbf{p}_{\mu}(\hbar) & \\
& =\sum_{g, n \geq 1} \frac{1}{n !} \hbar^{2 g-2+n} F_{g, n}^{E}(x, x, \ldots, x),
\end{aligned}
$$

which yields (6.2).

From the expansion formulas (5.12) and (4.5), together with the equality (6.2), we obtain

$$
\sum_{\mu}\left(\frac{|\mu| !}{\operatorname{dim} \mu}\right)^{1-\chi(B)} e^{\frac{1}{2} \mathbf{p}_{2}[\mu] \hbar} s_{\mu}(\mathbf{p}(\hbar))=\sum_{m=0}^{n} c_{m} e^{\frac{1}{2} m(m-1) \hbar}\left(\hbar^{1-\chi(B)} x\right)^{m}
$$

As explained in [26, Section 6] and also in [25], this equality is exactly the effect of the principal specialization of Lemma 6.1, which turns the summation over all partitions into the sums over just one-row partitions. We have thus determined the coefficients $c_{m}$ in the expansion (4.5). It is given by

$$
c_{m}=(m !)^{1-\chi(B)} .
$$

This completes the proof of (1.8). The convergence of the infinite series is obvious from the shape of (1.8) or (1.9), since

$$
\limsup _{m \rightarrow \infty}\left|(m !)^{1-\chi(B)} e^{\frac{1}{2} m(m-1) \hbar}\right|^{\frac{1}{m}}=0
$$

if $\operatorname{Re}(\hbar)<0$.

The expansion (1.8) allows us to derive the quantum curve-type equation (1.4). Since

$$
\begin{aligned}
{\left[\hbar^{1-\chi(B)} x e^{\hbar x \frac{d}{d x}}\left(\frac{d}{d x} x\right)^{1-\chi(B)}\right](m !)^{1-\chi(B)} e^{\frac{1}{2} m(m-1) \hbar}\left(\hbar^{1-\chi(B)} x\right)^{m} } & \\
= & ((m+1) !)^{1-\chi(B)} e^{\frac{1}{2} m(m+1) \hbar}\left(\hbar^{1-\chi(B)} x\right)^{m+1}
\end{aligned}
$$


for every $m \geq 0$, we have

$$
\left(1-\hbar^{1-\chi(B)} x e^{\hbar x \frac{d}{d x}}\left(\frac{d}{d x} x\right)^{1-\chi(B)}\right) Z^{B}(x, \hbar)=1 .
$$

By differentiating (6.5) we obtain (1.4).

Lemma 6.2. Let

$$
\begin{aligned}
& P=\hbar x \frac{d}{d x}\left(1-\hbar^{1-\chi(B)} x e^{\hbar x \frac{d}{d x}}\left(\frac{d}{d x} x\right)^{1-\chi(B)}\right) \\
& Q=\frac{\partial}{\partial \hbar}-\frac{1}{2}\left(x \frac{\partial}{\partial x}\right)^{2}+\left(\frac{1}{2}-\frac{1-\chi(B)}{\hbar}\right) x \frac{\partial}{\partial x} .
\end{aligned}
$$

Then

$$
[P, Q]=-\frac{1}{\hbar} P
$$

Proof. We first note that $Q$ commutes with $x \frac{d}{d x}$ and $\frac{d}{d x} x$. Since

$$
\begin{aligned}
& {\left[\hbar^{1-\chi(B)} x e^{\hbar x \frac{d}{d x}}, Q\right]} \\
& =\left[\hbar^{1-\chi(B)} x e^{\hbar x \frac{d}{d x}}, \frac{\partial}{\partial \hbar}\right]+\left[\hbar^{1-\chi(B)} x,-\frac{1}{2}\left(x \frac{\partial}{\partial x}\right)^{2}+\left(\frac{1}{2}-\frac{1-\chi(B)}{\hbar}\right) x \frac{\partial}{\partial x}\right] e^{\hbar x \frac{d}{d x}} \\
& =-(1-\chi(B)) \hbar^{-\chi(B)} x e^{\hbar x \frac{d}{d x}}-\hbar^{1-\chi(B)} x^{2} \frac{d}{d x} e^{\hbar x \frac{d}{d x}} \\
& \quad+\hbar^{1-\chi(B)} x^{2} \frac{d}{d x} e^{\hbar x \frac{d}{d x}}+\frac{1}{2} \hbar^{1-\chi(B)} x e^{\hbar x \frac{d}{d x}}-\hbar^{1-\chi(B)}\left(\frac{1}{2}-\frac{1-\chi(B)}{\hbar}\right) x e^{\hbar x \frac{d}{d x}} \\
& =0,
\end{aligned}
$$

(1.7) follows from

$$
0=\left[\frac{1}{\hbar} P, Q\right]=\frac{1}{\hbar}[P, Q]+\left[\frac{1}{\hbar}, Q\right] P=\frac{1}{\hbar}[P, Q]+\frac{1}{\hbar^{2}} P .
$$

\section{SEMI-CLASSICAL LIMIT}

The semi-classical analysis of the operators $P$ and $Q$ are performed in the following way. Suppose our counting problem had genus 0 contributions $F_{0, n}^{B}\left(x_{1}, \ldots, x_{n}\right)$. Then we define

$$
S_{m}(x):=\sum_{2 g-2+n=m-1} \frac{1}{n !} F_{g, n}^{B}(x, \ldots, x)
$$

as before, and consider a formal expression

$$
\bar{Z}^{B}(x, \hbar)=e^{\sum_{m=0}^{\infty} \hbar^{m-1} S_{m}(x)}=e^{\frac{1}{\hbar} S_{0}(x)+S_{1}(x)} Z^{B}(x, \hbar) .
$$

Let us introduce a variable $u$ such that $x=e^{u}$, and regard the coefficients $S_{m}$ as functions in $u$. Since

we have

$$
x \frac{d}{d x}=\frac{d}{d u}
$$

$$
P=\hbar \frac{d}{d u}\left(1-\hbar^{1-\chi(B)} e^{u} e^{\hbar \frac{d}{d u}}\left(1+\frac{d}{d u}\right)^{1-\chi(B)}\right)
$$


Then

$$
\begin{aligned}
e^{-S_{1}} e^{-\frac{1}{\hbar} S_{0}} P e^{\frac{1}{\hbar} S_{0}} e^{S_{1}} & =S_{0}^{\prime}-e^{u}\left(S_{0}^{\prime}\right)^{2-\chi(B)} e^{\frac{1}{\hbar}\left(S_{0}(u+\hbar)-S_{0}(u)\right)}+O(\hbar) \\
& =S_{0}^{\prime}-e^{u}\left(S_{0}^{\prime}\right)^{2-\chi(B)} e^{S_{0}^{\prime}}+O(\hbar),
\end{aligned}
$$

where $^{\prime}=\frac{d}{d u}=x \frac{d}{d x}$, and by $O(\hbar)$ we mean an operator whose application to the partition function $Z^{B}(x, \hbar)$ produces a function of order 1 or higher in $\hbar$. Now define a new variable

$$
y=S_{0}^{\prime} .
$$

Then the semi-classical limit $\hbar \rightarrow 0$ of (7.3) yields an equation

$$
y-x y^{2-\chi(B)} e^{y}=y\left(1-x y^{1-\chi(B)} e^{y}\right)=0,
$$

since $Z^{B}(x, 0)=1$. Note that this is exactly the total symbol of the operator $P$, where $\hbar x \frac{d}{d x}$ is represented by a commutative variable $y$. The second factor of (7.5) also recovers the Lambert curve (1.5).

Although the formal manipulation seems to work, however, we have to remember that we have derived the operator $P$ assuming the shape of the solution $Z^{B}(x, \hbar)$ as in (1.8). Since we are imposing

$$
P \bar{Z}^{B}(x, \hbar)=0,
$$

it forces that $y=0$, which makes (7.5) trivially correct.

On the other hand, since the kernel of $Q$ assums only the expansion (4.5), where the summation index $m$ can be negative, the semi-classical analysis of $Q$ does go through.

$$
\begin{aligned}
e^{-S_{1}} e^{-\frac{1}{\hbar} S_{0}} Q e^{\frac{1}{\hbar} S_{0}} e^{S_{1}} & e^{-S_{1}} e^{-\frac{1}{\hbar} S_{0}}\left[\frac{\partial}{\partial \hbar}-\frac{1}{2} \frac{\partial^{2}}{\partial u^{2}}+\left(\frac{1}{2}-\frac{1-\chi(B)}{\hbar}\right) \frac{\partial}{\partial u}\right] e^{\frac{1}{\hbar} S_{0}} e^{S_{1}} \\
= & -\frac{1}{\hbar^{2}}\left(S_{0}+\frac{1}{2}\left(S_{0}^{\prime}\right)^{2}+(1-\chi(B)) S_{0}^{\prime}\right) \\
& -\frac{1}{\hbar}\left(\frac{1}{2} S_{0}^{\prime \prime}-\frac{1}{2} S_{0}^{\prime}+S_{0}^{\prime} S_{1}^{\prime}+(1-\chi(B)) S_{1}^{\prime}\right)+O(1) .
\end{aligned}
$$

For the $\hbar \rightarrow 0$ limit to exist, we need

$$
\begin{aligned}
& S_{0}+\frac{1}{2}\left(S_{0}^{\prime}\right)^{2}+(1-\chi(B)) S_{0}^{\prime}=0, \\
& \frac{1}{2} S_{0}^{\prime \prime}-\frac{1}{2} S_{0}^{\prime}+S_{0}^{\prime} S_{1}^{\prime}+(1-\chi(B)) S_{1}^{\prime}=0 .
\end{aligned}
$$

From (7.7) we obtain

$$
S_{0}=-\frac{1}{2} y^{2}-(1-\chi(B)) y,
$$

or equivalently,

$$
y=-(y+1-\chi(B)) \frac{d y}{d u} .
$$

Its solution is

$$
y+\log y^{1-\chi(B)}=-u+\text { const. }
$$

If we take the constant of integration to be 0 , then we obtain

$$
e^{u}=x=y^{\chi(B)-1} e^{-y},
$$


recovering (1.5). From (7.8) we have

$$
\frac{1}{2} y^{\prime}-\frac{1}{2} y+(y+1-\chi(B)) S_{1}^{\prime}=0,
$$

or equivalently

$$
\frac{d S_{1}}{d u}=-\frac{1}{2} \frac{1}{y+1-\chi(B)} \frac{d y}{d u}+\frac{1}{2} \frac{y}{y+1-\chi(B)} \frac{d y}{d y} .
$$

Since we know $d u / d y$ from (7.10), we can integrate the above equation to obtain

$$
S_{1}=-\frac{1}{2} y+\frac{1}{2} \log (y+1-\chi(B))+\text { const. }
$$

The above derivation of the semi-classical limit of the operator $Q$ is valid if $Z^{B}(x, \hbar)$ contains negative powers of $\hbar$. If we assume the expansion (1.8), then such a situation occurs when the base curve $B$ satisfies $\chi(B)>1$. Indeed, our formulas (7.9) and (7.11) agree with those of [26, Section 5] for $B=\mathbb{P}^{1}$ with $y=z$, and [3, Section 6] for $B=\mathbb{P}^{1}[a]$ with $y=z^{a}$.

Acknowledgement. X.L. received the China Scholarship Council grant CSC-2010811063, which allowed him to conduct research at the Department of Mathematics, University of California, Davis. He is also supported by the National Science Foundation of China grants No.11201477, 11171175, and the Chinese Universities Scientific Fund No.2011JS041. The research of M.M. is supported by NSF grant DMS-1104734. A.S. received support from the U.S. Government.

\section{REFERENCES}

[1] M. Aganagic, R. Dijkgraaf, A. Klemm, M. Mariño, and C. Vafa, Topological Strings and Integrable Hierarchies, arXiv:hep-th/0312085, Commun. Math. Phys. 261, 451-516 (2006).

[2] G. Borot, B. Eynard, M. Mulase and B. Safnuk, Hurwitz numbers, matrix models and topological recursion, Journal of Geometry and Physics 61, 522-540 (2011).

[3] V. Bouchard, D. Hernández Serrano, X. Liu, and M. Mulase, Mirror symmetry for orbifold Hurwitz numbers, arXiv: 1301.4871.

[4] V. Bouchard, A. Klemm, M. Mariño, and S. Pasquetti, Remodeling the B-model, Commun. Math. Phys. 287, 117-178 (2008).

[5] V. Bouchard and M. Mariño, Hurwitz numbers, matrix models and enumerative geometry, Proc. Symposia Pure Math. 78, 263-283 (2008).

[6] R. Dijkgraaf, Mirror symmetry and elliptic curves, in "The Moduli Space of Curves," Progress in Mathematics vol. 129, Dijkgraaf et al., editors, 149-162, Birkhäuser 1995.

[7] R. Dijkgraaf, L. Hollands, and P. Sułkowski, Quantum curves and D-modules, Journal of High Energy Physics arXiv:0810.4157, 1-58 (2009).

[8] R. Dijkgraaf, L. Hollands P. Sułkowski, and C. Vafa, Supersymmetric gauge theories, intersecting branes and free Fermions, Journal of High Energy Physics arXiv:0709.4446, (2008).

[9] R. Dijkgraaf and C. Vafa, Two Dimensional Kodaira-Spencer Theory and Three Dimensional Chern-Simons Gravity, arXiv:0711.1932 [hep-th] (2007).

[10] N. Do, O. Leigh, and P. Norbury, Orbifold Hurwitz numbers and Eynard-Orantin invariants, arXiv:1212.6850 [math.AG] (2012).

[11] O. Dumitsrescu, M. Mulase, A. Sorkin and B. Safnuk, The spectral curve of the Eynard-Orantin recursion via the Laplace transform, arXiv:1202.1159 [math.AG] (2012).

[12] T. Ekedahl, S. Lando, M. Shapiro, A. Vainshtein, Hurwitz numbers and intersections on moduli spaces of curves, Invent. Math. 146, 297-327 (2001).

[13] B. Eynard, M. Mulase and B. Safnuk, The Laplace transform of the cut-and-join equation and the BouchardMariño conjecture on Hurwitz numbers, Publications of the Research Institute for Mathematical Sciences 47, 629-670 (2011).

[14] B. Eynard and N. Orantin, Invariants of algebraic curves and topological expansion, Communications in Number Theory and Physics 1, 347-452 (2007).

[15] B. Eynard and N. Orantin, Computation of open Gromov-Witten invariants for toric Calabi-Yau 3-folds by topological recursion, a proof of the BKMP conjecture, arXiv:1205.1103v1 [math-ph] (2012). 
[16] I.P. Goulden, A differential operato for symmetric functions and the combinatorics of multiplying transpositions, Trans. A.M.S., 344, 421-440 (1994).

[17] I.P. Goulden and D.M. Jackson, Transitive factorisations into transpositions and holomorphic mappings on the sphere, Proc. A.M.S., 125, 51-60 (1997).

[18] T. Graber and R. Vakil, Hodge integrals and Hurwitz numbers via virtual localization, Compositio Math. 135, 25-36 (2003).

[19] S. Gukov and P. Sułkowski, A-polynomial, B-model, and quantization, arXiv:1108.0002v1 [hep-th] (2011).

[20] A. Hurwitz, Über Riemann'sche Flächen mit gegebene Verzweigungspunkten, Mathematische Annalen 39, 1-66 (1891).

[21] M. Kaneko and D. Zagier, A generalized Jacobi theta function and quasi-modular forms, in "The Moduli Space of Curves," Progress in Mathematics vol. 129, Dijkgraaf et al., editors, 165-172, Birkhäuser 1995.

[22] M. Kazarian, KP hierarchy for Hodge integrals, arXiv:0809.3263 (2008).

[23] M. Kazarian, S. Lando, An algebro-geometric proof of Witten's conjecture, J. Amer. Math. Soc. 20, 1079-1089 (2007).

[24] M. Mariño, Open string amplitudes and large order behavior in topological string theory, J. High Energy Physics 0803-060, 1-33 (2008).

[25] M. Mulase, S. Shadrin, and L. Spitz, The spectral curve and the Schrödinger equation of double Hurwitz numbers and higher spin structures, arXiv:1301.5580, (2013).

[26] M. Mulase and P. Sułkowski, Spectral curves and the Schrödinger equations for the Eynard-Orantin recursion, arXiv:1210.3006, (2012).

[27] M. Mulase and J. Yu, A generating function of the number of homomorphisms from a surface group into a finite group, arXiv:math.QA/0209008, (2002).

[28] M. Mulase and N. Zhang, Polynomial recursion formula for linear Hodge integrals, Communications in Number Theory and Physics 4, 267-294 (2010).

[29] A. Okounkov, Toda equations for Hurwitz numbers, Math. Res. Lett. 7, 447-453 (2000).

[30] A. Okounkov and R. Pandharipande, Gromov-Witten theory, Hurwitz numbers, and matrix models, I, Proc. Symposia Pure Math. 80, 325-414 (2009).

[31] A. Okounkov and R. Pandharipande, Gromov-Witten theory, Hurwitz theory, and completed cycles, Ann. Math. 163, 517-560 (2006).

[32] R. Vakil, Harvard Thesis 1997.

[33] J. Zhou, Hodge integrals, Hurwitz numbers, and symmetric groups, preprint, arXiv:math/0308024, (2003).

[34] J. Zhou, Quantum Mirror Curves for $\mathbb{C}^{3}$ and the Resolved Confiold, arXiv:1207.0598v1, (2012).

Department of Applied Mathematics, China Agricultural University, Beijing, 100083, China, and Department of Mathematics, University of California, Davis, CA 95616-8633, U.S.A.

E-mail address: xjliu@cau.edu.cn

Department of Mathematics, University of California, Davis, CA 95616-8633, U.S.A.

E-mail address: mulase@math.ucdavis.edu

Department of Mathematics, University of California, Davis, CA 95616-8633, U.S.A.

E-mail address: azsorkin@math.ucdavis.edu 